Original Article

\title{
Effect of two load escalation methods using a supine ergometer on the cardiorespiratory system and lower limb muscle activity
}

\author{
Masahiro Noguchi, RPT, PhD ${ }^{1)^{*}}$, Kentaro SASAKI, RPT, PhD ${ }^{1)}$ \\ 1) Faculty of Health Science, Kinjo University: 1200 Kasama-machi, Hakusan-shi, Ishikawa 924-8511, \\ Japan
}

\begin{abstract}
Purpose] This study aimed to determine the effects of two load escalation methods, such as pedal weight increasing method and pedal speed raising method using a supine ergometer, on the cardiorespiratory system and lower limb muscle activity. [Participants and Methods] The study included 20 healthy adult males (age: $21 \pm 1$ years). Two different parameters were modulated during exercise intervention. Pedal load progression was applied in the load group $(28.9 \mathrm{~N}, 72.3 \mathrm{~N}, 101.3 \mathrm{~N})$, and pedal rate progression was applied in the speed group $(60 \mathrm{rpm}, 80 \mathrm{rpm}, 100 \mathrm{rpm})$. Each group performed 5 minutes of exercise at their respective level of progression, for a total duration of 15 minutes. [Results] Oxygen consumption, carbon dioxide output, and minute ventilation were significantly increased as exercise intensity increased in both groups. Significant differences in muscle activity were found between the highest exercise intensities (101.3 $\mathrm{N}$ and $100 \mathrm{rpm})$. The activities of the biceps femoris and gastrocnemius were significantly higher with exercise at $100 \mathrm{rpm}$ than at $101.3 \mathrm{~N}$. [Conclusion] Increasing the number of revolutions per minute may significantly increase the activity of the lower limb muscles at comparable exercise intensities, despite the low work load.

Key words: Supine ergometer, Electromyography, Exercise intensity
\end{abstract}

(This article was submitted Apr. 10, 2019, and was accepted May 30, 2019)

\section{INTRODUCTION}

The rehabilitation of patients receiving dialysis plays an important role in the extension of healthy life expectancy or improvement of life prognosis improvement. However, the consecutive rehabilitation intervention is difficult due to worsening of physical conditioning or time constraint in patients on dialysis. Patients on dialysis are instructed to lie down for a long period of time during dialysis for 3-4 hours at a time, 2-3 days a week. As a result, a vicious circle is created in which chronic inactivity results in lowering physical fitness and physical function, which leads to further inactivity. Johansen et al. ${ }^{1)}$ reported that the patients on hemodialysis have muscle atrophy, and they found an association between muscle atrophy and frailty. The survey in patients with sarcopenia showing age-related muscle decrease shows that sarcopenia is associated with low survival rates ${ }^{2,3}$. Moreover, a study reported that $22.2 \%$ of outpatients on hemodialysis have a sarcopenia, and it was found in $48 \%$ of the patients $>70$ years. Continuous exercise (increase of physical activity) is necessary to prevent or improve the decline of the physical strength of patients on dialysis and to put an end to the vicious circle. Currently, efforts to exercise during dialysis are increasing from the viewpoint of continuity and efficiency of exercise. Exercise during dialysis has benefits and advantages. It can easily reduce the time of inactivity during bedtime dialysis, and because it can be performed at regular dialysis time, continuing the exercise is easy. As a result, improvements of oxygen uptake and walking ability have been reported ${ }^{4)}$.

Regarding exercise during dialysis, various attempts such as ergometer exercise (supine position ergometer exercise) and

*Corresponding author. Masahiro Noguchi (E-mail: noguchi@kinjo.ac.jp)

(C2019 The Society of Physical Therapy Science. Published by IPEC Inc.

(c) (1) $\odot$ This is an open-access article distributed under the terms of the Creative Commons Attribution Non-Commercial No Deriva-

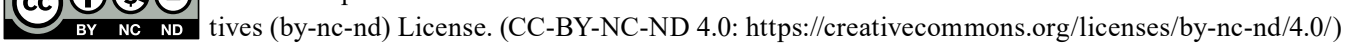


resistance training have been reported. However, no regulation such as exercise intensity setting method or detailed exercise method has been established. The Japanese Society of Renal Rehabilitation introduced an ergometer instrument (Terasu Ergo, Showa Denki, Osaka, Japan) that can be used in the lying position. However, the method of setting exercise intensity remained unclear with guidelines. As a concrete formulation of exercise intensity, there is a method to increase the weight of the pedal and to use heart rate or subjective exercise intensity as a guide. However, in patients on dialysis susceptible to muscle weakness, if the pedal is heavy, fatigue of the lower limb occurs, and continuing exercise becomes difficult. The exercise intensity of the ergometer is determined by the product of the weight of the pedal and the number of rotations. Therefore, if increasing the weight of the pedal is difficult, the exercise intensity can be adjusted by changing the rotation speed. However, only a few such attempts have been reported.

In a study of oxygen uptake and heart rate during pedaling with varying pedaling speeds with constant workload, even in exercise of the same workload, an increased oxygen intake or heart rate by pedal rate has been reported ${ }^{5}$. In a study that attempted to incorporate the pedal revolution number to estimate the oxygen intake during exercise, the accuracy of oxygen uptake estimation has been reported to be improved by incorporating the revolution number into the estimation equation ${ }^{6}$. In the survey of the effect of muscle activity and circadian rhythm during ergometer exercise for cyclist, the time to peak torque was delayed when the pedal speed was increased ${ }^{7)}$. Thus, although the exercise intensity setting of the ergometer in the supine position is mainly adjusted by the weight of the pedal presently, there is a possibility that adjustment by the pedal speed may be more effective in the future. However, only a few studies investigating the pedal speed in the supine position ergometer exercise have been conducted. To elucidate the setting of the exercise intensity during dialysis, studies clarifying the influence of the pedal weight and rotation number on the human body in the supine position ergometer motion are considered necessary. In this study, the exercise intensity for supine ergometer exercise was focused on the method of setting, and investigated how the difference in exercise intensity increment method affects the human body.

This study aimed to clarify the influence of cardiorespiratory system and lower limb muscle activity by two load escalation methods such as pedal weight increasing method and pedal speed raising method.

\section{PARTICIPANTS AND METHODS}

The study participants included 20 healthy adult males. The mean age was $21 \pm 1$ years, the mean height was $170.3 \pm$ $5.2 \mathrm{~cm}$, the mean weight was $63.4 \pm 7.5 \mathrm{~kg}$, and the mean body mass index was $21.9 \pm 2.6 \mathrm{~kg} / \mathrm{m}^{2}$. The participants were recruited by posting details of this study on the university bulletin board. We decided to exclude applicants who met the exclusion criteria described later; however, none of the applicants met the exclusion criteria in this study. Exclusion criteria of this study were a person who had respiratory and circulatory diseases in the past and are still affected at the time of exercise, a person who have disadvantage for ergometer exercise with injury of the lower limb or pain, and a person who cannot provide consent to participate In this research, although the influence of the weight of the pedal and the number of rotations of the pedal were experimented, it is necessary to further study the setting method such as changing the number of rotations finely with each weight. The research protocol was explained to the participants who provided their informed consent prior to the commencement of the study. This study was approved by the Kinjo University Research Ethics Committee (approval number No. 27-09), and the research was conducted in compliance with the terms of the Declaration of Helsinki.

Concerning the exercise intervention, patients performed ergometer exercise while supine on a bed. The "Terasu ergo" was used as a load-variable ergometer (Showa Denki Co. Ltd., Osaka, Japan). This instrument can manually adjust the pedal load amount to seven stages: $20 \mathrm{~W}, 30 \mathrm{~W}, 40 \mathrm{~W}, 50 \mathrm{~W}, 60 \mathrm{~W}, 65 \mathrm{~W}$, and $70 \mathrm{~W}$. This load indication (W) represents the weight of the pedal, and there is correspondence of the factual pedal load amount as shown in Table 1.

The expired gas (oxygen intake $\left[\mathrm{VO}_{2}\right]$, carbon dioxide $\left[\mathrm{VCO}_{2}\right]$, and expiratory minute volume [VE]) and vital data (heart rate [HR], systolic blood pressure [SBP], and diastolic blood pressure [DBP]) were measured using an analysis of expired gas system (AT-1100, Anima Co., Ltd., Tokyo, Japan), a cardiography equipment (ECG-1550, Nihon Kohden Co., Ltd., Tokyo, Japan), and a blood pressure monitoring device (Tango, Santech Medical Inc., NC, USA). The rating of perceived exertion (RPE) during exercise was measured based on physical sensations including breathing difficulty and leg fatigue using the Borg Scale. The muscle activity of the lower limb muscle associated with cycling was measured using a surface electromyograph (EM-401, Noraxon Co., Ltd., AZ, USA). The test muscles were five external muscles: the vastus lateralis, the rectus femoris, the long head of the biceps femoris, the tibialis anterior, and the lateral head of the gastrocnemius muscle. The method of attaching the electromyogram conformed to the method of Perotto ${ }^{8}$. The electrodes of the vastus lateralis muscle were attached over the lateral aspect of the thigh, one handbreadth above the patella. The electrodes of the rectus femoris muscle were attached on the anterior aspect of the thigh, midway between the superior border of the patella and the anterior superior iliac spine. The electrodes of the long head of the biceps femoris muscle were attached at the midpoint of a line between the fibula head and the ischial tuberosity. The electrode of the tibialis anterior muscle was attached at four fingerbreadths below the tibial tuberosity and one fingerbreadth lateral to the tibial crest. The electrode

Table 1. Load indication (W) and the factual pedal load

\begin{tabular}{cc}
\hline $\begin{array}{c}\text { Load indication } \\
(\mathrm{W})\end{array}$ & $\begin{array}{c}\text { Pedal load } \\
(\mathrm{N})\end{array}$ \\
\hline 20 & 28.9 \\
30 & 43.4 \\
40 & 57.9 \\
50 & 72.3 \\
60 & 86.8 \\
65 & 94.0 \\
70 & 101.3 \\
\hline
\end{tabular}




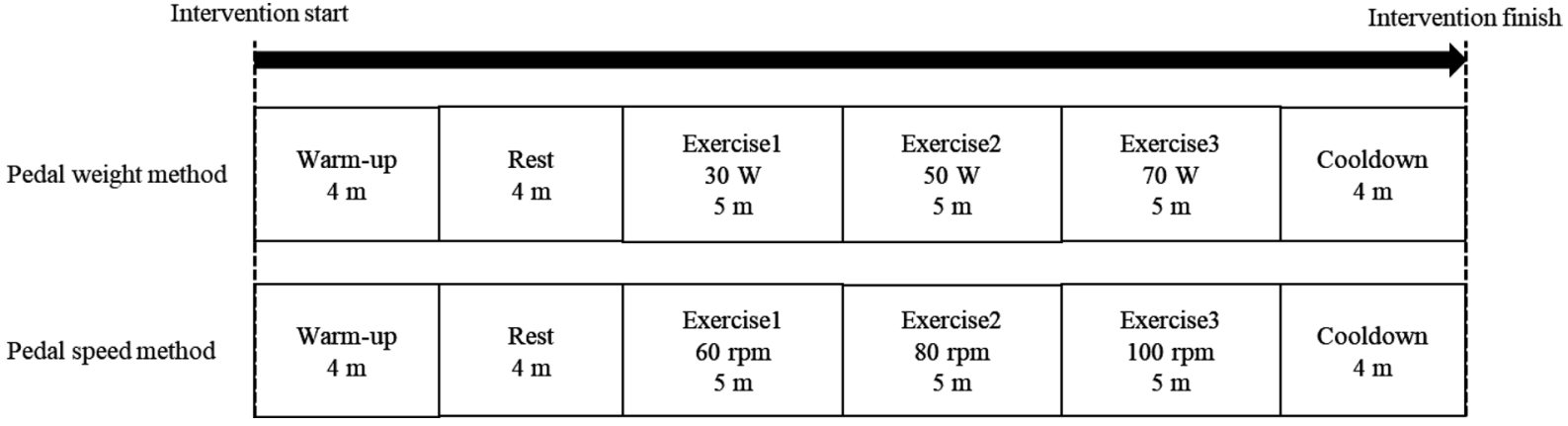

Fig. 1. Schematic representation of the exercise interventions.

of the lateral head of the gastrocnemius muscle was attached at one hand breadth below the popliteal crease on the lateral mass of the calf.

Two types of exercise load were used in this study: pedal weight load increase without changing pedal speed and pedal speed increase without changing pedal weight. Moreover, two types of exercise load were used in this study such as pedal weight load gradual increase method without changing pedal speed (pedal weight method) and pedal speed gradual increase method without changing pedal weight (pedal speed method). The pedal weight method was increased in three levels: $30 \mathrm{~W}$ $(43.4 \mathrm{~N}), 50 \mathrm{~W}(72.3 \mathrm{~N})$, and $70 \mathrm{~W}(101.3 \mathrm{~N})$ at $60 \mathrm{rpm}$. The pedal speed method was increased in three levels: $60 \mathrm{rpm}$, $80 \mathrm{rpm}$, and $100 \mathrm{rpm}$ with $43.4 \mathrm{~N}$. Each exercise level was performed for 5 minutes, and the total exercise time was 15 minutes. The participants performed warm-up exercises for 4 minutes with $28.9 \mathrm{~N}, 60 \mathrm{rpm}$ in both methods. To eliminate the effect of each exercise intervention, one exercise intervention was performed at least 1 day after the other was performed. Exercise protocols consisted of a resting period of 4 minutes; warm-up, 4 minutes; exercise, 15 minutes (each step for 5 minutes); and cooldown, 4 minutes, totaling 27 minutes (Fig. 1). The order of pedal weight method or pedal speed method was random.

The ergometer used in this study cannot record the distance. Therefore, the factual distance was calculated by the number of rotations during exercise and pedal circumference. Furthermore, the workload ( $\mathrm{J}$ ) during exercise was calculated using the factual distance and pedal weight. The crank arm of the supine position ergometer equipment was $11.5 \mathrm{~cm}$, and the whole circumference of the pedal was $0.72 \mathrm{~m}$. By multiplying this circumference with the number of revolutions performed in 5 minutes, the actual driving distance was obtained. Since the pedal weight method fixed the rotation speed at $60 \mathrm{rpm}$, the driving distance was $216 \mathrm{~m}$ in all stages. In the pedal speed method, the cycling distances were $216 \mathrm{~m}, 288 \mathrm{~m}$, and $360 \mathrm{~m}$ at $60 \mathrm{rpm}, 80 \mathrm{rpm}$, and $100 \mathrm{rpm}$, respectively. The workload during exercise was calculated by multiplying this cycling distance with the pedal weight $(\mathrm{N})$. The results showed that the workload increased to $9374.4 \mathrm{~J}, 15616.8 \mathrm{~J}$, and $21880.8 \mathrm{~J}$ in the pedal weight method and $9374.4 \mathrm{~J}, 12499.2 \mathrm{~J}$, and $15624.0 \mathrm{~J}$ in the pedal speed method. Data on the expired gas, the vitals, and muscle activity were compared between each exercise method, and the influence on the physical activity was determined by each method. An average value of data $\left(\mathrm{VO}_{2}, \mathrm{VCO}_{2}, \mathrm{VE}, \mathrm{HR}, \mathrm{SBP}, \mathrm{DBP}\right)$ in the last 3 minutes was calculated as a representative value of rest and three levels of exercise. Similarly, the average value of the muscle activity of each muscle in the last 3 minutes of each exercise stage was considered as the representative value. In RPE, the peak value of breathing difficulty was used, and leg fatigue was used as the representative value.

The $\mathrm{VO}_{2}, \mathrm{VCO}_{2}, \mathrm{VE}, \mathrm{HR}, \mathrm{SBP}$, and DBP during rest periods and three levels of exercise between pedal weight method and the pedal speed method were compared using repeated measure analysis of variance and multiple comparison technique. RPE and muscle activity of the three levels of exercise were compared using Wilcoxon signed-rank test, which corrected the significance level using the Shaffer's method. In addition, the exercise intensity of each exercise intervention was set to three levels of low intensity, moderate intensity, and high intensity, and the 2-exercise intensity between each group of the pedal weight method and the pedal speed method was compared. The $\mathrm{VO}_{2}, \mathrm{VCO}_{2}, \mathrm{VE}, \mathrm{HR}, \mathrm{SBP}$, and DBP were compared using paired t-test, and RPE and muscle activity were compared using Wilcoxon signed-rank test. The 5\% level was considered as statistically significant. All statistical analyses were performed using SPSS, version 19.0, software (IBM Corp., Armonk, NY, USA).

\section{RESULTS}

The workload during exercise was calculated from the results of this study (Table 2). Table 3 shows the expired gas and vital data during resting periods and exercise. For the expired gas, $\mathrm{VO}_{2}, \mathrm{VCO}_{2}$, and $\mathrm{VE}$ were found to have significant differences among the four groups of rest and exercise in both methods. Moreover, significant differences were observed in $\mathrm{VO} 2, \mathrm{VCO} 2$, and $\mathrm{VE}$ among rest periods and all three exercise levels in both pedal load methods $(\mathrm{p}<0.01)$. In the comparison between each exercise intensity stage of pedal weight method and pedal speed method, significant differences were observed 
Table 2. Cycling distance and workload

\begin{tabular}{lccccc}
\hline Intensity & Pedal weight method (W) & Pedal speed (rpm) & Pedal load (N) & Cycling distance (m) & Workload (J) \\
\hline Low & 30 & 60 & 43.4 & 216 & $9,374.4$ \\
Moderate & 50 & 60 & 72.3 & 216 & $15,616.8$ \\
High & 70 & 60 & 101.3 & 216 & $21,880.8$ \\
\hline Intensity & Pedal speed method (rpm) & Pedal weight (W) & Pedal load (N) & Cycling distance (m) & Workload (J) \\
\hline Low & 60 & 30 & 43.4 & 216 & $9,374.4$ \\
Moderate & 80 & 30 & 43.4 & 288 & $12,499.2$ \\
High & 100 & 30 & 43.4 & 360 & $15,624.0$ \\
\hline
\end{tabular}

Table 3. Expired gas data and vital data

\begin{tabular}{|c|c|c|c|c|c|}
\hline & & Resting & Low intensity & Moderate intensity & High intensity \\
\hline \multirow{2}{*}{$\begin{array}{l}\mathrm{VO}_{2} \\
\quad(\mathrm{ml} / \mathrm{kg})\end{array}$} & Pedal weight method & $338.64 \pm 85.41$ & $649.15 \pm 126.92^{* *}$ & $829.39 \pm 131.14^{* * \# \#}$ & $1056.32 \pm 121.65^{* * \# \# \S \S}$ \\
\hline & Pedal speed method & $364.51 \pm 83.97$ & $676.46 \pm 132.35^{* *}$ & $895.55 \pm 134.66^{* * \# \# \dagger}$ & $1175.53 \pm 159.50^{* * \# \# \S \S \dagger \dagger}$ \\
\hline \multirow{2}{*}{$\begin{array}{l}\mathrm{VCO}_{2} \\
\quad(\mathrm{ml} / \mathrm{kg})\end{array}$} & Pedal weight method & $313.80 \pm 83.75$ & $555.36 \pm 91.20^{* *}$ & $737.08 \pm 98.97^{* * \# \#}$ & $979.65 \pm 98.12^{* * \# \# \S \S}$ \\
\hline & Pedal speed method & $345.06 \pm 57.07^{\dagger}$ & $588.45 \pm 78.32^{* * \dagger}$ & $817.36 \pm 89.52^{* *+\dagger}$ & $1116.37 \pm 139.93^{* * \# \# \S \S \dagger \dagger}$ \\
\hline \multirow{2}{*}{$\begin{array}{l}\text { VE } \\
\qquad(\mathrm{ml})\end{array}$} & Pedal weight method & $12.28 \pm 2.82$ & $19.95 \pm 3.60^{* *}$ & $25.30 \pm 4.15^{* * \# \#}$ & $32.50 \pm 4.71^{* * \# \# \S \S}$ \\
\hline & Pedal speed method & $13.60 \pm 2.71^{\dagger}$ & $21.05 \pm 3.51^{* *}$ & $27.65 \pm 3.91^{* * \#+\dagger \dagger}$ & $37.54 \pm 6.24^{* * \# \# \S \S \dagger \dagger}$ \\
\hline \multirow{2}{*}{$\begin{array}{l}\text { SBP } \\
\qquad(\mathrm{mmHg})\end{array}$} & Pedal weight method & $119.05 \pm 11.87$ & $129.77 \pm 10.59 * *$ & $137.42 \pm 10.92^{* * \# \#}$ & $151.89 \pm 14.00^{* * \# \# \S \S}$ \\
\hline & Pedal speed method & $118.65 \pm 13.37$ & $125.75 \pm 14.11^{* *}$ & $138.28 \pm 11.59^{* * \# \#}$ & $149.49 \pm 15.53^{* * \# \# \S \S}$ \\
\hline \multirow{2}{*}{$\begin{array}{l}\text { DBP } \\
\quad(\mathrm{mmHg})\end{array}$} & Pedal weight method & $65.85 \pm 9.45$ & $66.01 \pm 9.42$ & $66.92 \pm 8.72$ & $68.64 \pm 10.26$ \\
\hline & Pedal speed method & $59.20 \pm 9.12^{\dagger \dagger}$ & $64.36 \pm 8.35^{*}$ & $64.03 \pm 8.70^{*}$ & $65.10 \pm 9.56^{*}$ \\
\hline \multirow{2}{*}{$\begin{array}{l}\text { HR } \\
\quad(\mathrm{bpm}) \\
\end{array}$} & Pedal weight method & $74.00 \pm 13.27$ & $93.48 \pm 9.92^{* *}$ & $104.97 \pm 11.15^{* * \# \#}$ & $115.77 \pm 12.29^{* * \# \# \S \S}$ \\
\hline & Pedal speed method & $76.90 \pm 14.41$ & $97.30 \pm 10.98^{* *}$ & $107.83 \pm 9.33^{* * \# \#}$ & $120.16 \pm 12.17^{* * \# \# \S \S \dagger}$ \\
\hline
\end{tabular}

Values are presented as mean $\pm \mathrm{SD}$.

$\mathrm{VO}_{2}$ : oxygen intake; $\mathrm{VCO}_{2}$ : carbon dioxide intake; VE: expiratory minute volume; HR: heart rate; SBP: systolic blood pressure; DBP: diastolic blood pressure.

Comparison with resting data: ${ }^{* *} \mathrm{p}<0.01,{ }^{*} \mathrm{p}<0.05$; comparison with low intensity: ${ }^{\# *} \mathrm{p}<0.01$; comparison with moderate intensity:

$\S \S_{p}<0.01$; Comparison of pedal weight method and pedal speed method: ${ }^{\dagger} p<0.01,{ }^{\dagger} p<0.05$.

Table 4. Rating of perceived exertion results

\begin{tabular}{llcc}
\hline & & Pedal weight method & Pedal speed method \\
\hline \multirow{2}{*}{ Low intensity } & Breathing difficulty & $9.1 \pm 1.5$ & $7.8 \pm 1.3^{* *}$ \\
& Leg fatigue & $10.4 \pm 1.6$ & $9.5 \pm 1.7^{*}$ \\
Moderate intensity & Breathing difficulty & $10.8 \pm 2.4$ & $9.7 \pm 2.0^{*}$ \\
& Leg fatigue & $13.2 \pm 1.8$ & $12.5 \pm 1.7$ \\
High intensity & Breathing difficulty & $12.3 \pm 3.0$ & $11.7 \pm 2.6$ \\
& Leg fatigue & $14.9 \pm 1.9$ & $14.7 \pm 1.8$ \\
\hline
\end{tabular}

Values are presented as mean $\pm \mathrm{SD}$.

Comparison of pedal weight method and pedal speed method: ${ }^{*} \mathrm{p}<0.05$.

between moderate intensity $(\mathrm{p}<0.01)$ and high intensity $(\mathrm{p}<0.01)$ of $\mathrm{VO}_{2}$ and $\mathrm{VE}$ and low intensity $(\mathrm{p}<0.05)$, moderate intensity $(\mathrm{p}<0.01)$, and high intensity $(\mathrm{p}<0.01)$ of $\mathrm{VCO}_{2}$.

In the vital data, HR and SBP were found to have significant differences among the four sets of rest and exercise in both methods ( $\mathrm{p}<0.01$ ). Significant differences were observed between rest period and $60 \mathrm{rpm}, 80 \mathrm{rpm}$, and $100 \mathrm{rpm}$ of DBP in pedal speed method $(\mathrm{p}<0.05)$. In the comparison between the pedal weight method and pedal speed method, significant differences were found in DBP during rest period $(\mathrm{p}<0.01)$ and in HR during high-intensity exercise $(\mathrm{p}<0.05)$, and a significant high HR during high-intensity exercise was observed in the pedal speed method.

Table 4 shows the results of RPE. In the comparison between the pedal weight method and pedal speed method, significant differences were observed in breathing difficulty and leg fatigue during low-intensity exercise $(\mathrm{p}<0.01, \mathrm{p}<0.05$, respectively) and in breathing difficulty during moderate-intensity exercise $(p<0.05)$. Even in high-intensity exercise, RPE tends to be lower in the pedal speed method, and strong fatigue was felt more in the lower limbs than in the chest at all strengths. 
Table 5. Results of the percent of the maximum voluntary contraction (\%MVC)

\begin{tabular}{llccc}
\hline Muscles & Method & Low intensity & Moderate intensity & High intensity \\
\hline Vastus lateralis & Pedal weight & $15.17 \pm 19.07$ & $21.24 \pm 25.91^{* *}$ & $29.84 \pm 43.06^{* * \#}$ \\
& Pedal speed & $9.45 \pm 7.28$ & $14.49 \pm 10.19^{* *}$ & $18.60 \pm 11.92^{* * \# \#}$ \\
Rectus femoris & Pedal weight & $9.48 \pm 13.14$ & $12.75 \pm 15.64^{* *}$ & $18.15 \pm 22.43^{* * \# \#}$ \\
& Pedal speed & $6.96 \pm 8.78$ & $9.37 \pm 11.17^{* *}$ & $12.12 \pm 15.29^{* * \# \# \dagger}$ \\
Long head of the & Pedal weight & $4.77 \pm 4.30$ & $7.10 \pm 5.25^{* *}$ & $9.37 \pm 7.26^{* * \# \#}$ \\
biceps femoris & Pedal speed & $5.42 \pm 3.43$ & $8.50 \pm 4.91^{* *}$ & $15.24 \pm 7.97^{* * \# \# \dagger}$ \\
Lateral head of the & Pedal weight & $8.37 \pm 5.67$ & $9.82 \pm 5.98^{*}$ & $11.99 \pm 7.65^{* * \#}$ \\
gastrocnemius & Pedal speed & $11.79 \pm 16.39$ & $23.25 \pm 46.69^{* *}$ & $27.98 \pm 36.41^{* * \# \# \dagger}$ \\
Tibialis anterior & Pedal weight & $9.43 \pm 8.75$ & $13.16 \pm 10.38^{* *}$ & $15.46 \pm 12.49^{* * \# \#}$ \\
& Pedal speed & $7.61 \pm 5.77$ & $11.85 \pm 7.44^{* *}$ & $19.61 \pm 15.45^{* * \# \#}$ \\
\hline
\end{tabular}

Values are presented as mean $\pm \mathrm{SD}$.

Comparison with low intensity: ${ }^{* *} \mathrm{p}<0.01,{ }^{*} \mathrm{p}<0.05$; Comparison with moderate intensity: ${ }^{\#} \mathrm{p}<0.01$; Comparison of pedal weight method and pedal speed method: ${ }^{\dagger} \mathrm{p}<0.01,{ }^{\dagger} \mathrm{p}<0.05$.

Table 5 shows the results of the muscle activity. All test muscles such as the vastus lateralis muscle, the rectus femoris muscle, the long head of the biceps femoris, the tibialis anterior muscle, and the lateral head of gastrocnemius muscle were found to have significant differences among the three exercise levels ( $<<0.01, p<0.05$ only in moderate-intensity exercise of the lateral head of the gastrocnemius muscle). In the comparison between the pedal weight method and pedal speed method, significant differences were observed in high intensity of the rectus femoris muscle, the long head of the biceps femoris, and the lateral head of gastrocnemius muscle $(\mathrm{p}<0.01)$. A significantly high activity was observed in the rectus femoris muscle in the pedal speed method, and the biceps femoris and the lateral head of the gastrocnemius muscle tended to be significantly high in activity in the pedal weight method.

\section{DISCUSSION}

The expired gas analysis, HR, and blood pressure increased significantly upon increasing the exercise intensity in both the pedal weight method and the pedal speed method, and both the pedal weight and the pedal speed were suggested to be useful for adjusting the exercise intensity. Currently, in clinical practice, exercise intensity adjustment for the supine position ergometer exercise is often performed by the pedal weight method. However, this result indicates that the exercise load can be even increased using the pedal speed method. In particular, the pedal speed method, it was possible to increase the exercise intensity by about 3 METs or $40 \mathrm{bpm}$ at maximum compared to the resting state when the ergometer exercise was performed on the bed in the supine position. Therefore, even for patients with unstable sitting and standing positions, higher intensity exercise can be prescribed, and this would be useful for future reference.

The workload was calculated from the pedal weight and the factual distance in both methods. The workload in the pedal weight method was approximately $15,616 \mathrm{~J}$ in moderate intensity and approximately $21,880 \mathrm{~J}$ in high intensity. In the pedal speed method, the workload was approximately $12,499 \mathrm{~J}$ in moderate intensity and approximately $15,624 \mathrm{~J}$ in high intensity and was higher in the pedal weight method. In particular, this time, the moderate intensity of the pedal weight method and the high intensity of the pedal speed method have almost equivalent workloads. Pedal weight step-up was predicted to increase the workload and the burden on the body. However, in the results of expired gas data and vital data as shown in Table 3, the pedal speed method was significantly higher in the moderate intensity, $\mathrm{VO}_{2}, \mathrm{VCO}_{2}$, and $\mathrm{VE}$ and $\mathrm{HR}$ in the high intensity than in the pedal weight method. This suggests that an increase in pedal speed may prompt more ventilation and circulation despite a low workload in the supine position ergometer motion. In a study of changing the number of rotation by constant work rate using sitting bicycle ergometer, increasing of the number of rotation increases the oxygen uptake and heart rate than increasing of the pedal weight ${ }^{5}$. In the report of Miyoshi et al. ${ }^{9}$, muscle activity increased by pedal weight in the supine ergometer. Kato et al. ${ }^{10)}$ reported that the exercise in supine ergometer has a low oxygen uptake and significantly high peak torque for leg compared with recumbent ergometer. Additionally, in the current study, the muscle activity of the long head of the biceps femoris and the lateral head of gastrocnemius muscle increased via increasing the number of rotation, and the workload was significantly higher: $15,624 \mathrm{~J}$ exercise against $21,880 \mathrm{~J}$ exercise. The rectus femoris muscle had low muscle activity in the pedal weight method than in the pedal speed method. This result indicates that the increment of the pedal speed increases the muscle activity amount of the lower limb than the increase of the pedal weight, regardless of the workload during exercise. Furthermore, the exercise load, which increases the rotation speed, mainly utilized the flexor muscle group of the knee joint to increase the rotation speed. In contrast, it was suggested that the exercise load, which increases the weight of the pedal, mainly utilizes the extensor group of the knee joint. In particular, if the pedal speed is increased by increasing the rotation speed, strong force is required for pulling up the pedal, resulting in increased muscle activity of the flexion muscle group of the knee joint. According to Silva et al., lower limb movement during cycling is affected by biarticular muscles such as the 
quadriceps femoris and biceps femoris muscle ${ }^{11}$. This study also suggests that the influence of the rectus femoris muscle, biceps femoris, and gastrocnemius muscle on the biarticular muscle produced significant effects. When considering the exercise prescription of the supine position ergometer exercise, further studies are necessary to investigate the combination of the pedal load amount and the rotational speed.

The expired gas data of the pedal speed method have shown significantly higher values despite the low workload in moderate intensity and high intensity compared to the pedal weight method. However, RPE of the pedal speed method tended to decrease compared to the pedal weight method, and significant difference was observed between the results of expiration gas and RPE. This result indicates that there is no significant influence on RPE even if the oxygen uptake amount increases by increasing the pedal speed. In the survey of increasing the number of rotations for the 7-bicycle athlete, increased cardiac output, decreased arterial-mixed venous oxygen difference, and increased oxygen uptake in connection with increasing 70 , 90 , and $110 \mathrm{rpm}$ of the number of rotations occurred ${ }^{12}$. Furthermore, the number of pedal rotations has been reported to be an important determinant of oxygen uptake ${ }^{6}$. Therefore, the number of pedal rotations has a great influence on oxygen uptake and is thought to play an important role in gradual increase of load during exercise. Furthermore, the increased number of the pedal rotations may be an effective exercise intensity setup, because muscle activity around the knee joint increases during exercise of low rotations such as 60 or $80 \mathrm{rpm}$. However, we could not directly clarify which of the two methods-pedal weight method and pedal speed method - is superior for setting the exercise intensity. Therefore, it is necessary to unify the workload of the two exercise intensity gradual increase methods, and further evaluate the effects of the use of these methods.

The results of this study suggested that increased number of pedal rotations increased significant muscle activity of flexion muscles around the knee joint. However, the participants of this study were healthy adult men, and these results cannot be used for patients on dialysis directly. Patients on dialysis have longer bed rest than healthy people, and the influence of muscle atrophy and physical strength decreases is great ${ }^{3,4)}$. A more careful exercise load setting is required for patients on dialysis who have lower muscle strength of the lower limbs. According to Koga et al. ${ }^{13)}$, the maximum Watt, the maximum oxygen intake, and the maximum heart rate in the supine position are significantly lower, and vigorous athletic activity in the supine position has a risk of increasing oxygen transport and oxygen use. Therefore, taking together the results of this study, it is suggested that adjustment be made based on the number of revolutions with less than moderate exercise intensity. In this research, although the influence of the weight of the pedal and the number of rotations of the pedal were investigated, it is necessary to further study the setting method, such as by changing the number of rotations with each weight.

It was possible to increase muscle activity while maintaining exercise intensity moderately at 60 to 80 pedal rotations. Adjusting exercise at this speed while keeping pedal weight constant may allow more effective intervention. Future clinical studies are necessary to clarify whether this result is effective also for patients on dialysis.

\section{Funding and Conflict of interest}

This research has no funding source.

\section{REFERENCES}

1) Johansen KL, Shubert T, Doyle J, et al.: Muscle atrophy in patients receiving hemodialysis: effects on muscle strength, muscle quality, and physical function Kidney Int, 2003, 63: 291-297. [Medline] [CrossRef]

2) Isoyama N, Qureshi AR, Avesani CM, et al.: Comparative associations of muscle mass and muscle strength with mortality in dialysis patients. Clin J Am Soc Nephrol, 2014, 9: 1720-1728. [Medline] [CrossRef]

3) Matsubara Y, Matsumoto T, Aoyagi Y, et al.: Sarcopenia is a prognostic factor for overall survival in patients with critical limb ischemia. J Vasc Surg, 2015, 61: 945-950. [Medline] [CrossRef]

4) Matsuzawa R, Hoshi K, Yoneki K, et al.: Exercise training in elderly people undergoing hemodialysis: a systematic review and meta-analysis. Kidney Int Rep, 2017, 2: 1096-1110. [Medline] [CrossRef]

5) Hirano M, Shindo M, Mishima S, et al.: Effects of 2 weeks of low-intensity cycle training with different pedaling rates on the work rate at lactate threshold. Eur J Appl Physiol, 2015, 115: 1005-1013. [Medline] [CrossRef]

6) Formenti F, Minetti AE, Borrani F: Pedaling rate is an important determinant of human oxygen uptake during exercise on the cycle ergometer. Physiol Rep, 2015, 3: e12500. [Medline] [CrossRef]

7) Bessot N, Moussay S, Clarys JP, et al.: The influence of circadian rhythm on muscle activity and efficient force production during cycling at different pedal rates. J Electromyogr Kinesiol, 2007, 17: 176-183. [Medline] [CrossRef]

8) Perotto AO: Anatomical guide for the electromyographer: the limbs and trunk. 5th ed. Illinois: Springfield, 2011, pp $194-257$.

9) Miyoshi K, Ohira M, Kanno Y: Diffrences in muscle activities of healthy males in bicycle ergometer exercise in the supine and sitting positions (Japanese). Rigakuryoho Kagaku, 2016, 31: 829-833. [CrossRef]

10) Kato M, Tsutsumi T, Yamaguchi T, et al.: Characteristics of maximum performance of pedaling exercise in recumbent and supine positions. J Sports Sci Med, 2011, 10: 491-497. [Medline]

11) da Silva JC, Tarassova O, Ekblom MM, et al.: Quadriceps and hamstring muscle activity during cycling as measured with intramuscular electromyography. Eur J Appl Physiol, 2016, 116: 1807-1817. [Medline] [CrossRef]

12) Gotshall RW, Bauer TA, Fahrner SL: Cycling cadence alters exercise hemodynamics. Int J Sports Med, 1996, 17: 17-21. [Medline] [CrossRef]

13) Koga S, Shiojiri T, Shibasaki M, et al.: Kinetics of oxygen uptake during supine and upright heavy exercise. J Appl Physiol 1985, 1999, 87: 253-260. [Medline] [CrossRef] 